УДК 514.764.2

\title{
Трехмерные локально симметрические (псевдо)римановы многообразия с векторным кручением и нулевым тензором кривизны ${ }^{*}$
}

\author{
П.Н. Клепиков, Е.Д. Родионов, О.П. Хромова
}

Алтайский государственный университет (Барнаул, Россия)

\section{Three-Dimensional Locally Symmetric (Pseudo)Riemannian Manifolds with Vectorial Torsion and Zero Curvature Tensor}

\author{
P.N. Klepikov, E.D. Rodionov, O.P. Khromova \\ Altai State University (Barnaul, Russia)
}

Метрическая связность с векторным кручением (также известная как полусимметрическая связность) является одной из трех основных связностей, описанных Э. Картаном. Данная связность играет важную роль в случае двумерных поверхностей, так как при этом любая метрическая связность является связностью с векторным кручением.

К. Яно была доказана важная теорема о связи конформных деформаций и метрических связностей с векторным кручением. А именно: риманово многообразие допускает метрическую связность с векторным кручением, тензор кривизны которой равен нулю, тогда и только тогда, когда оно является конформно плоским. Таким образом, возникает задача об изучении (псевдо)римановых многообразий с метрической связностью с векторным кручением, тензор кривизны которых равен нулю.

Данная работа посвящена решению поставленной задачи в случае трехмерных локально симметрических многообразий. Кроме того, приводится математическая модель, позволяющая вычислять компоненты тензора кривизны метрической связности с векторным кручением в случае локально однородных (псевдо)римановых многообразий.

Ключевые слова: (псевдо)римановое многообразие, метрическая связность с векторным кручением, локально однородные многообразия, тензор кривизны.

DOI 10.14258/izvasu(2019)4-13

1. Введение, определения и постановка задачи. Пусть $(M, g)$ - (псевдо)риманово многообразие. Определим на данном многообразии

* Работа выполнена при поддержке РФФИ (грант: № 1831-00033 мол_а).
A metric connection with vectorial torsion (also known as a semi-symmetric connection) is one of the three main connections described by E. Cartan. This connection plays an important role in the case of two-dimensional surfaces since, in this case, any metric connection is a connection with vectorial torsion.

K. Yano proved an important theorem on the connection of conformal deformations and metric connections with vectorial torsion. Namely, a Riemannian manifold admits a metric connection with vectorial torsion, the curvative tensor of which is zero, if and only if it is conformally flat. Thus, the problem of studying (pseudo)Riemannian manifolds with metric connection with vectorial torsion, the curvature tensor of which is zero, is arisen.

This paper is devoted to solving the problem in the case of three-dimensional locally symmetric manifolds. In addition, a mathematical model is presented that allows one to calculate the components of the curvature tensor of a metric connection with vectorial torsion in the case of locally homogeneous (pseudo)Riemannian manifolds.

Key words: (pseudo)Riemannian manifold, metric connection with vectorial torsion, locally homogeneous manifolds, curvature tensor.

метрическую связность $\nabla$ с помощью формулы

$$
\nabla_{X} Y=\nabla_{X}^{g} Y+g(X, Y) V-g(V, Y) X,
$$

где $V$ - некоторое фиксированное векторное поле, $X$ и $Y-$ произвольные векторные поля, $\nabla^{g}-$ связность Леви-Чивита. Связность $\nabla$ является одной из трех основных связностей, описанных 
Э. Картаном в работе [1], и называется метрической связностью с векторным кручением, или полусимметрической связностью (с точностью до направления).

Данная связность играет важную роль в случае двумерных поверхностей, так как в этом случае любая метрическая связность является связностью с векторным кручением [1]. В работах [2-7] изучаются различные аспекты метрических связностей с векторным кручением.

Важная теорема о связи конформных деформаций и метрических связностей с векторным кручением была доказана К. Яно в работе [8].

Теорема. Риманово многообразие допускает метрическую связность с векторным кручением, тензор кривизны которой равен нулю, тогда и только тогда, когда оно является конформно плоским.

Таким образом, возникает задача об изучении (псевдо)римановых многообразий с метрической связностью с векторным кручением, тензор кривизны которых равен нулю. Данная работа посвящена решению поставленной задачи в случае трехмерных локально симметрических многообразий.

2. Предварительные сведения. Тензор кривизны метрической связности $\nabla$ с векторным кручением определяется аналогично общему случаю равенством

$$
R(X, Y) Z=\nabla_{Y} \nabla_{X} Z-\nabla_{X} \nabla_{Y} Z+\nabla_{[X, Y]} Z .
$$

Отметим, что, в отличие от случая связности Леви-Чивита, в данном случае тензор кривизны не обязан удовлетворять алгебраическому тождеству Бьянки. Однако верна

Теорема $[\mathbf{9}, \mathbf{1 0}]$. Пусть $(M, g)-($ псевдо)риманово многообразие с метрической связностью с векторным кручением. Тогда тензор кривизны удовлетворяет алгебраическому тождеству Бьянки

$$
R(X, Y) Z+R(Y, Z) X+R(Z, X) Y=0
$$

тогда и только тогда, когда 1-форма $\pi$ замкнута (т.е. $d \pi=0)$, где $\pi(X)=g(X, V)$ для любого векторного поля $X$ на $M$.

Исследование кривизны трехмерных локально однородных (псевдо)римановых многообразий основывается на следующей теореме, которая была доказана в римановом случае в работе [11], а в лоренцевом - в [12].

Теорема. Пусть $(M, g)$ - трехмерное локально однородное (псевдо)риманово многообразие.

Тогда либо $(M, g)$ является локально симметричным (относительно связности Леви-Чивита), либо оно локально изометрично трехмерной группе Ли с левоинвариантной (псевдо)римановой метрикой.
Следующий классификационный результат для случая трехмерных (псевдо)римановых локально симметричных пространств был получен в $[11,12]$.

Теорема. Трехмерное локально симметричное (псевдо)риманово многообразие $(M, g)$ локально изометрично

1. (псевдо)римановой пространственной форме $\mathbb{R}^{3}, \mathbb{S}^{3}$ или $\mathbb{H}^{3}$ (с нулевой, положительной или отрицательной секционной кривизной соответственно), или

2. прямому произведению $\mathbb{S}^{2} \times \mathbb{R}$ или $\mathbb{H}^{2} \times \mathbb{R}$, или

3. многообразию Уокера (т.е. многообразию c параллельным изотропным распределением) $c$ лоренцевой метрикой $g$, которое допускает локальную систему координат $\left(u_{1}, u_{2}, u_{3}\right)$ такую, что метрический тензор имеет вид

$$
g=\left(\begin{array}{ccc}
0 & 0 & 1 \\
0 & \varepsilon & 0 \\
1 & 0 & u_{2}^{2} \alpha+u_{2} \beta\left(u_{3}\right)+\xi\left(u_{3}\right)
\end{array}\right),
$$

где $\varepsilon= \pm 1, \alpha \in \mathbb{R}, \beta$ и $\xi-$ произвольные гладкие функции.

Для удобства вычислений мы используем представление локально однородного пространства $M=G / H$ в виде алгебр Ли (подробнее см. [13]). Пусть $\mathfrak{g}$ - алгебра Ли группы изометрий $G, \mathfrak{h}$ - алгебра Ли подгруппы изотропии $H, \mathfrak{m}$ - дополнение к $\mathfrak{h}$ до алгебры $\mathfrak{g}$. Пусть $\operatorname{dim} \mathfrak{h}=h$ и $\operatorname{dim} \mathfrak{m}=m$. Зафиксируем базис $\left\{e_{1}, \ldots, e_{h}, u_{1}, \ldots, u_{m}\right\}$ алгебры $\mathfrak{g}$, где $\left\{e_{i}\right\}$ и $\left\{u_{i}\right\}$ базисы $\mathfrak{h}$ и $\mathfrak{m}$ соответственно.

В работе [14] получена классификация трехмерных локально однородных (псевдо)римановых пространств. Далее мы будем использовать нумерацию из этой работы. В частности, из данной классификации следует

Теорема 1. Пусть $M=G / H-$ трехмерное локально однородное многообразие, допускающее локально симметричную (псевдо)риманову метрику. Тогда в алгебре Ли группы $G$ существует базис $\left\{e_{1}, \ldots, e_{h}, u_{1}, u_{2}, u_{3}\right\}$, где $\left\{e_{i}\right\}$ и $\left\{u_{i}\right\}-$ базисы $\mathfrak{h}$ и $\mathfrak{m}$ соответственно, такой, что скобки Ли имеют вид, приведенный в таблице.

Таблица для каждого случая также содержит вид инвариантного метрического тензора.

Опишем математическую модель, позволяющую вычислять компоненты тензора кривизны для локально однородных (псевдо)римановых многообразий с метрической связностью с инвариантным векторным кручением. Положим,

$$
\begin{gathered}
{\left[u_{i}, u_{j}\right]_{\mathfrak{m}}=c_{i j}^{k} u_{k}, \quad\left[u_{i}, u_{j}\right]_{\mathfrak{h}}=C_{i j}^{k} e_{k},} \\
{\left[e_{i}, u_{j}\right]_{\mathfrak{m}}=\bar{c}_{i j}^{k} u_{k},}
\end{gathered}
$$


Трехмерные локально симметрические (псевдо)римановы пространства

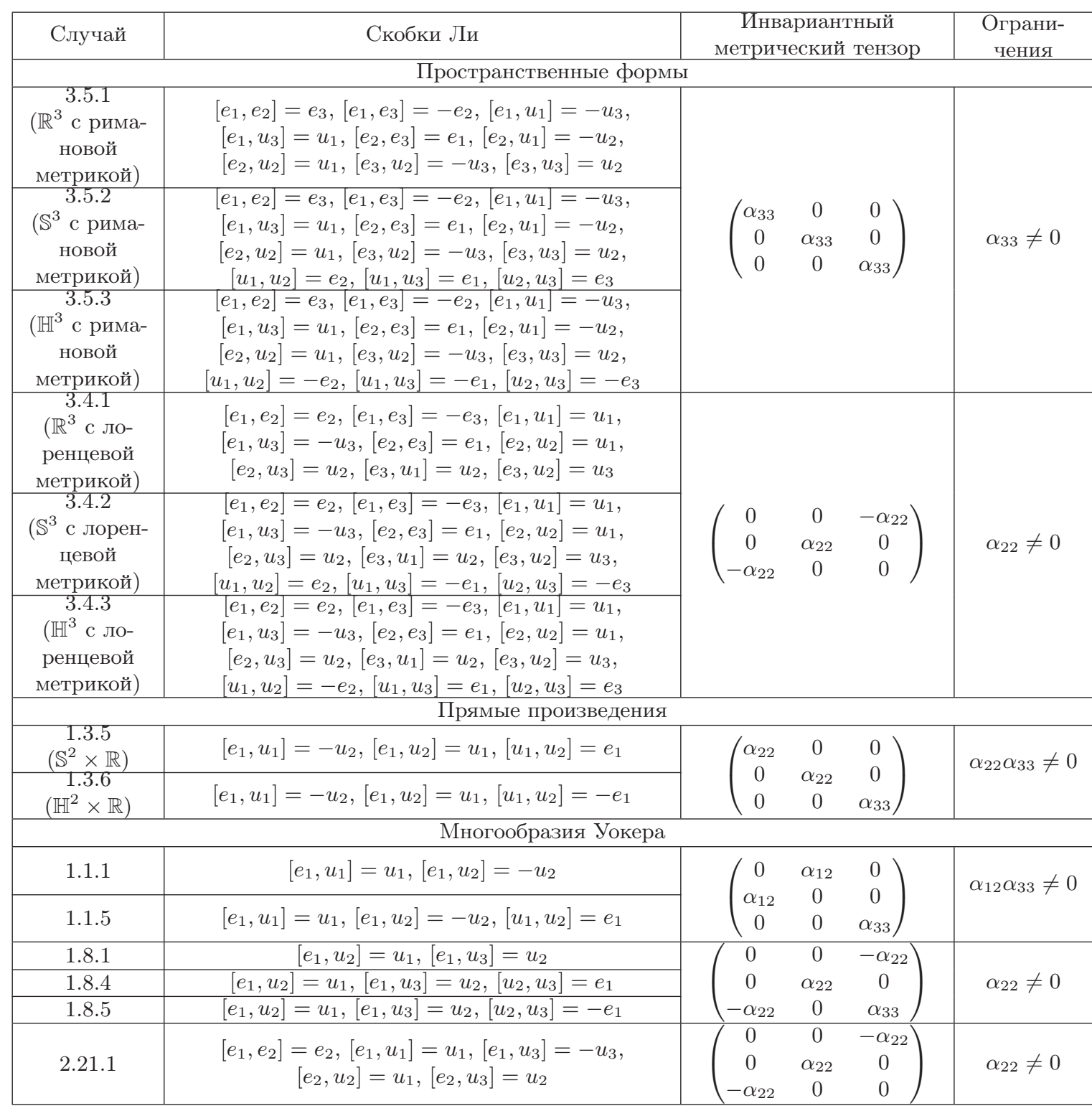

где $c_{i j}^{k}, C_{i j}^{k}$ и $\bar{c}_{i j}^{k}-$ массивы соответствующих размеров.

Представление изотропии $\psi$ на базисных векторах $\mathfrak{h}$ задается равенством $\left(\psi_{i}\right)_{j}^{k}=\left(\psi\left(e_{i}\right)\right)_{j}^{k}=$ $\bar{c}_{i j}^{k}$, тогда условие инвариантности метрического тензора $g$ имеет вид:

$$
\left(\psi_{i}\right)^{t} \cdot g+g \cdot \psi_{i}=0, \quad i=1, \ldots, h,
$$

где $\left(\psi_{i}\right)^{t}$ - транспонированная матрица.

Компоненты связности Леви-Чивита $\nabla^{g}$ выражаются через структурные константы и компоненты метрического тензора:

$$
\left(\Gamma^{g}\right)_{i j}^{k}=\frac{1}{2}\left(c_{i j}^{k}+g^{s k} c_{s j}^{l} g_{i l}+g^{s k} c_{s i}^{l} g_{j l}\right),
$$

$$
\left(\bar{\Gamma}^{g}\right)_{i j}^{k}=\frac{1}{2} \bar{c}_{i j}^{k}-\frac{1}{2} g^{s k} \bar{c}_{i s}^{l} g_{j l}
$$

где $\nabla_{u_{i}}^{g} u_{j}=\left(\Gamma^{g}\right)_{i j}^{k} u_{k}, \nabla_{e_{i}}^{g} u_{j}=\left(\bar{\Gamma}^{g}\right)_{i j}^{k} u_{k}$ и $\left\{g^{i j}\right\}-$ матрица, обратная к матрице $\left\{g_{i j}\right\}$.

Пусть инвариантный вектор $V \in \mathfrak{m}$, тогда компоненты метрической связности $\nabla$ с векторным кручением (1) задаются равенствами:

$$
\Gamma_{i j}^{k}=\left(\Gamma^{g}\right)_{i j}^{k}+g_{i j} V^{k}-V^{s} g_{s j} \delta_{i}^{k}, \quad \bar{\Gamma}_{i j}^{k}=\left(\bar{\Gamma}^{g}\right)_{i j}^{k},
$$

где $\nabla_{u_{i}} u_{j}=\Gamma_{i j}^{k} u_{k}, \nabla_{e_{i}} u_{j}=\bar{\Gamma}_{i j}^{k} u_{k}$.

Компоненты тензора кривизны $R$ можно вычислить с помощью следующей формулы:

$$
R_{i j k s}=\left(\Gamma_{i k}^{l} \Gamma_{j l}^{p}-\Gamma_{j k}^{l} \Gamma_{i l}^{p}+c_{i j}^{l} \Gamma_{l k}^{p}+C_{i j}^{l} \bar{\Gamma}_{l k}^{p}\right) g_{p s}
$$


или

$$
\begin{gathered}
R_{i j k s}=\frac{1}{4}\left(c_{i k}^{r}+g^{t r} c_{t k i}+g^{t r} c_{t i k}+2 g_{i k} V^{r}-2 \delta_{i}^{r} V_{k}\right) \\
\left(c_{j r s}+c_{s r j}+c_{s j r}+2 g_{j r} V_{s}-2 g_{j s} V_{r}\right)- \\
-\frac{1}{4}\left(c_{j k}^{r}+g^{t r} c_{t k j}+g^{t r} c_{t j k}+2 g_{j k} V^{r}-2 \delta_{j}^{r} V_{k}\right) \\
\left(c_{i r s}+c_{s r i}+c_{s i r}+2 g_{i r} V_{s}-2 g_{i s} V_{r}\right)- \\
-\frac{1}{2} c_{i j}^{r}\left(c_{r k s}+c_{s k r}+c_{s r k}+2 g_{r k} V_{s}-2 g_{r s} V_{k}\right)+ \\
+\frac{1}{2} C_{i j}^{l}\left(\bar{c}_{l k s}-\bar{c}_{l s k}\right),
\end{gathered}
$$

где $V_{k}=V^{s} g_{s k}, c_{i j k}=c_{i j}^{s} g_{s k}, \bar{c}_{i j k}=\bar{c}_{i j}^{s} g_{s k}$.

Докажем лемму, которую далее будем использовать для упрощения выкладок.

Лемма. Пусть $(\mathrm{G} / \mathrm{H}, \mathrm{g})$ - локально однородное (псевдо)риманово многообразие с метрической связностью с инвариантным векторным кручением. Тогда условие $d \pi=0$ равносильно

$$
g\left(V,[X, Y]_{\mathfrak{m}}\right)=0, \quad \forall X, Y \in \mathfrak{g} ;
$$

либо в базисе

$$
V^{i} g_{i j} c_{k t}^{j}=0, \quad V^{i} g_{i j} \bar{c}_{s t}^{j}=0 .
$$

Здесь $i, j, k, t=1, \ldots, m ; s=1, \ldots, h$.

Доказательство. Заметим, что

$$
\begin{aligned}
2 d \pi(X, Y) & =X \pi(Y)-Y \pi(X)-\pi([X, Y])= \\
& =-2 \pi([X, Y])=-2 g([X, Y], V)= \\
& =-2 g\left([X, Y]_{\mathfrak{m}}+[X, Y]_{\mathfrak{h}}, V\right) .
\end{aligned}
$$

Т.к. $V \in \mathfrak{m}$ и $\mathfrak{m} \perp \mathfrak{h}$, получаем

$$
d \pi(X, Y)=-g\left([X, Y]_{\mathfrak{m}}, V\right) .
$$

Фиксируя некоторый базис $\left\{e_{1}, \ldots, e_{h}, u_{1}, \ldots, u_{m}\right\}$ алгебры $\mathfrak{g}$, из данного равенства получаем условие (2).

Следствием предыдущей леммы является

Теорема 2. Пусть $(\mathrm{G} / \mathrm{H}, \mathrm{g})$ - трехмерное локально симметрическое (псевдо)риманово многообразие с метрической связностью с инвариантным векторным кручением. Тогда, если выполнено условие $d \pi=0$, то

- в случаях 2.21.1, 3.4.1, 3.4.2, 3.4.3, 3.5.1, 3.5.2, 3.5.3 вектор $V$ равен нулю;

- в случаях 1.1.1, 1.1.5, 1.3.5, 1.3 .6 вектор $V$ имеет координаты $\left(0,0, V^{3}\right)$;

- в случаях 1.8.1, 1.8.4, 1.8 .5 вектор $V$ имеет координаты $\left(V^{1}, 0,0\right)$.

Доказательство. Приведем доказательство теоремы для случая 1.1.1, для остальных случаев теоремы 1 доказательство аналогично. В этом случае система уравнений (2) имеет вид

$$
V^{1} \alpha_{12}=0, \quad V^{2} \alpha_{12}=0 .
$$

Т.к. $\alpha_{12} \neq 0$ в силу ограничений на компоненты метрического тензора, то $V^{1}=V^{2}=0$.
3. Основной результат. Главным результатом данной работы является следующая

Теорема 3. Пусть $(\mathrm{G} / \mathrm{H}, \mathrm{g})$ - трехмерное локально симметрическое (псевдо)риманово многообразие с метрической связностью с инвариантным векторным кручением. Тогда если тензор кривизны равен нулю, то

- в случаях 1.1.1, 1.8.1, 2.21.1, 3.4.1, 3.5.1 вектор $V$ равен нулю;

- в случае 1.1 .5 вектор $V$ имеет координаты $\left(0,0, \pm \frac{1}{\sqrt{-\alpha_{12} \alpha_{33}}}\right)$

- в случае 1.3.5 вектор $V$ имеет координаты $\left(0,0, \pm \frac{1}{\sqrt{-\alpha_{22} \alpha_{33}}}\right)$ и метрика обязана быть лоренцевой;

- в случае 1.3.6 вектор $V$ имеет координаты $\left(0,0, \pm \frac{1}{\sqrt{\alpha_{22} \alpha_{33}}}\right)$ и метрика обязана быть римановой;

- в случае 1.8.4 вектор $V$ имеет координаты $\left( \pm \frac{1}{\alpha_{22}}, 0,0\right)$.

В случаях 1.8.5, 3.4.2, 3.4.3, 3.5.2, 3.5.3 тензор кривизны не может быть равен нулю.

Доказательство. Рассмотрим доказательство теоремы для случая 1.8.4, для остальных случаев теоремы 1 доказательство аналогично.

При равенстве нулю тензора кривизны тождество Бьянки автоматически выполняется, следовательно, необходимо $d \pi=0$. Тогда по теореме 2 вектор $V$ имеет координаты $\left(V^{1}, 0,0\right)$ и уравнение $R=0$ имеет вид

$$
\left(\left(V^{1} \alpha_{22}\right)^{2}-1\right) \alpha_{22}=0
$$

Так как $\alpha_{22} \neq 0$ в силу условий на компоненты метрического тензора, то $V^{1}= \pm \frac{1}{\alpha_{22}}$.

4. Заключение. В результате проведенных исследований построена математическая модель, которая позволяет вычислять компоненты тензора кривизны локально однородных (псевдо)римановых многообразий с метрической связностью с инвариантным векторным кручением. Кроме того, в работе доказан ряд структурных теорем. 


\section{Библиографический список}

1. Cartan E. Sur les variétés à connexion affine et la théorie de la relativité generalisée (deuxiéme partie) // Ann. Ecole Norm. Sup.1925. Vol. 42.

2. Muniraja G. Manifolds Admitting a Semi-Symmetric Metric Connection and a Generalization of Schur's Theorem // Int. J. Contemp. Math. Sci. 2008. Vol. 3. No 25.

3. Agricola I., Thier C. The Geodesics of Metric Connections with Vectorial Torsion // Annals of Global Analysis and Geometry. 2004. Vol. 26.

4. Murathan C., Özgür C. Riemannian manifolds with a semi-symmetric metric connection satisfying some semisymmetry conditions // Proceedings of the Estonian Academy of Sciences. 2008. Vol. 57. No 4.

5. Yilmaz H.B., Zengin F.Ö., Uysal. S.A. On a Semi Symmetric Metric Connection with a Special Condition on a Riemannian Manifold // European journal of pure and applied mathematics. 2011. Vol. 4. No 2.

6. Zengin F.Ö., Demirbağ S.A., Uysal. S.A., Yilmaz H.B. Some vector fields on a riemannian manifold with semisymmetric metric connection // Bulletin of the Iranian Mathematical Society. 2012. Vol. 38. No 2.
7. Agricola I., Kraus M. Manifolds with vectorial torsion // Dierential Geometry and its Applications. 2016. Vol. 46.

8. Yano K. On semi-symmetric metric connection // Revue Roumame de Math. Pure et Appliquees. 1970. Vol. 15.

9. Barua B., Ray A. Kr. Some properties of a semi-symmetric metric connection in a Riemannian manifold // Indian J. pure appl. Math. 1985. Vol. 16, No 7.

10. De U.C., De B.K. Some properties of a semi-symmetric metric connection on a Riemannian manifold // Istanbul Univ. Fen. Fak. Mat. Der. 1995. Vol. 54.

11. Sekigawa K. On some 3-dimensional curvature homogeneous spaces // Tensor N. S. 1977. Vol. 31.

12. Calvaruso G. Homogeneous structures on threedimensional Lorentzian manifolds // J. Geom. Phys. 2007. Vol. 57.

13. Хромова О. П. Применение пакетов символьных вычислений к исследованию оператора одномерной кривизны на нередуктивных однородных псевдоримановых многообразиях // Известия АлтГУ. 2017. № 1(93).

14. Можей Н.П. Когомологии трехмерных однородных пространств // Труды БГТУ. 2014. № 6. 